\title{
The Effect of Knee Flexion Angle on Contact Stress of Total Knee Arthroplasty
}

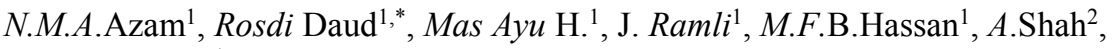 \\ M.A.H.M.Adib ${ }^{1}$ \\ ${ }^{1}$ Faculty of Mechanical Engineering, Universiti Malaysia Pahang, 26600 Pekan, Pahang, Malaysia \\ ${ }^{2}$ Faculty of Technical and Vocational, Universiti Pendidikan Sultan Idris, 35900 Tanjung Malim, \\ Perak, Malaysia
}

\begin{abstract}
The effect of flexion angle on contact stress of the knee joint still open to the debate since lack of proof shown that flexion angles does effect the contact stress of Total Knee Arthroplasty (TKA). Thus the aim of this study is to investigate the effect of different flexion angle on contact stress of TKA via finite element method. The TKA is simulated using ANSYS Workbench and the applied loads are 2200 N, $3200 \mathrm{~N}$ and 2800 N. The Finite element Analysis (FEA) results for maximum stress of current and proposed designed were then compared. For the new proposed design, the maximum stress for $15^{\circ}$ is $12.2 \mathrm{MPa}, 45^{\circ}$ is $23.6 \mathrm{MPa}$ and $60^{\circ}$ is $22.5 \mathrm{MPa}$ which is lower than current design. Thus, it can be concluded that the new proposed design better than current design in term of contact stress. While, the different flexion angle do gives an impact on the performance of the TKA.
\end{abstract}

\section{Introduction}

Loosening of the total knee arthroplasty joint is significantly correlated with the design. There is some reason that can make the artificial became loose. One is mechanical and is related to the fact that the knee joint bears a great deal of weight when a person is walking or running. It is unusual for the metal part of a knee implant to simply break. This part, however, is inserted into the upper part of the tibia, the larger of the two bones in the lower leg, after the surgeon has removed the upper surface of the tibia [1]. The bone tissue that receives the metal implant is softer than the bone that was removed, which means that the metal implant may sink into the softer bone and gradually loosen.

Two main design approaches for the bearing component of total knee arthroplasty are at the moment available on the market: the fixed one and the mobile one. In order to achieve the complex motions of the natural knee, fixed bearing total knee replacements are obliged to incongruent contacts. Small contact areas, in conjunctions with high joint loads, fatigue and delamination type wear of the polyethylene (PE). The knee implant design should take consideration of contact area stress of the knee joint same as applied to other joints such as hip or ankle [2-5].

The contact area distribution ratios decreased with increasing temperature, indicating that contact geometries become more circular at higher temperatures. The contact area

\footnotetext{
* Corresponding author: rosdidaud@ump.edu.my
} 
distribution ratios of most of the knees tested showed no clear change with flexion angle [6]. In a few cases, the ratios decreased as the flexion angle increased, indicating that contact geometries became more circular at $60^{\circ}$ than they were at smaller flexion angles [1]. Due to the effect of flexion angle on contact stress of the knee joint still open to the debate [6-8], thus the aim of this study is to investigate the effect of different flexion angle on contact stress of the TKA.

\section{Methodology}

The solid models of TKA as shown in Figure 1 and 2 were constructed by using SolidWorks Software 2015. By referring to the research of Villa et al. [9], the boundary condition included three different flexion angles of current knee implant was selected to be compared to the proposed design.

i) TKA $15^{\circ}$ of flexion with $2200 \mathrm{~N}$ load apply

ii) TKA $45^{\circ}$ of flexion with $3200 \mathrm{~N}$ load apply

iii) TKA $60^{\circ}$ of flexion with $2800 \mathrm{~N}$ load apply

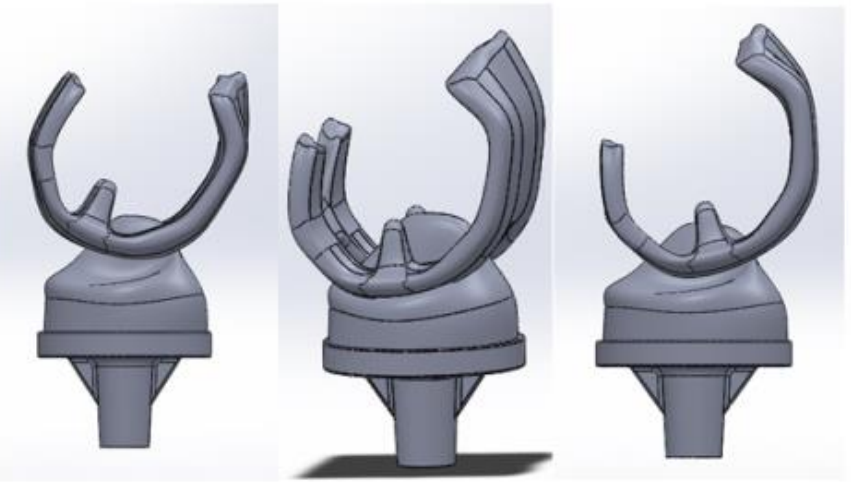

Fig. 1. The design of TKA $15^{\circ}, 45^{\circ}$ and $60^{\circ}$ of flexion angle as referred to the previous journal [9].

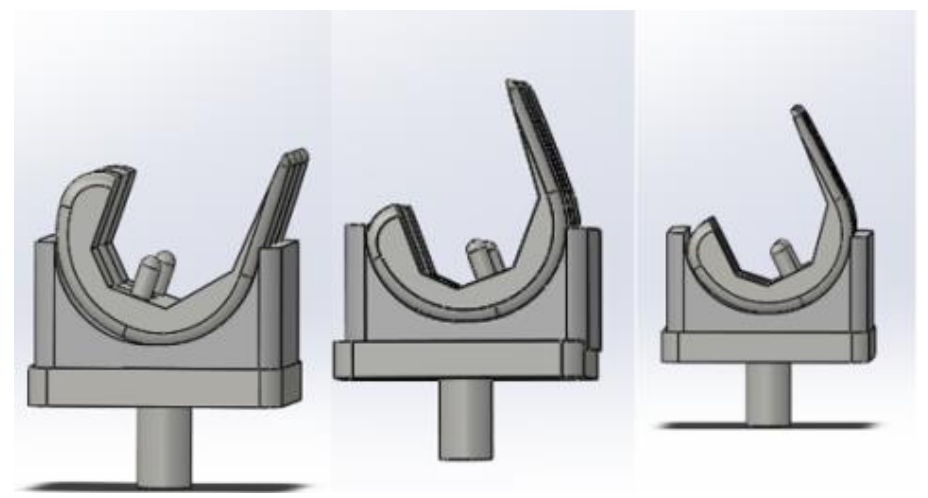

Fig. 2. The propose designs of TKA with $15^{\circ}, 45^{\circ}$ and $60^{\circ}$ of flexion angle.

In order to perform static structural of maximum stress test, as shown in Figure 3, the tibial tray was completely fixed in all flexion angle and an initial load of $2200 \mathrm{~N}$ was applied to the surface of polyethylene (mobile bearing of TKA) in flexion angle of $15^{\circ}$ and it was repeated with the different load of $3200 \mathrm{~N}$ for $45^{\circ}$ load of $2800 \mathrm{~N}$ for $60^{\circ}$. This FE model is 
considered verified when the FEA result obtained (as shown in Figure 4) is almost the same with the FEA result by Villa et. al. [9]. The proposed design of the total knee Arthroplasty is then analysed using this verified FE model with the same condition (flexion angles and load applied). Both proposed and current results of Finite Element Analysis (FEA) are compared to see either the proposed or the current design have the better performance.

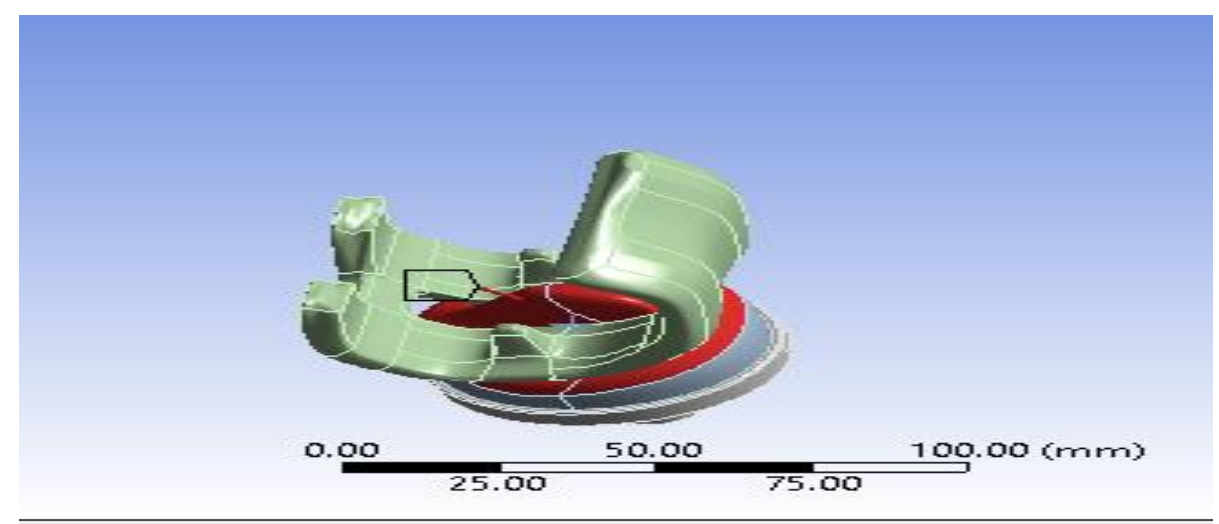

Fig. 3. Load applied on the TKA body at $15^{\circ}$

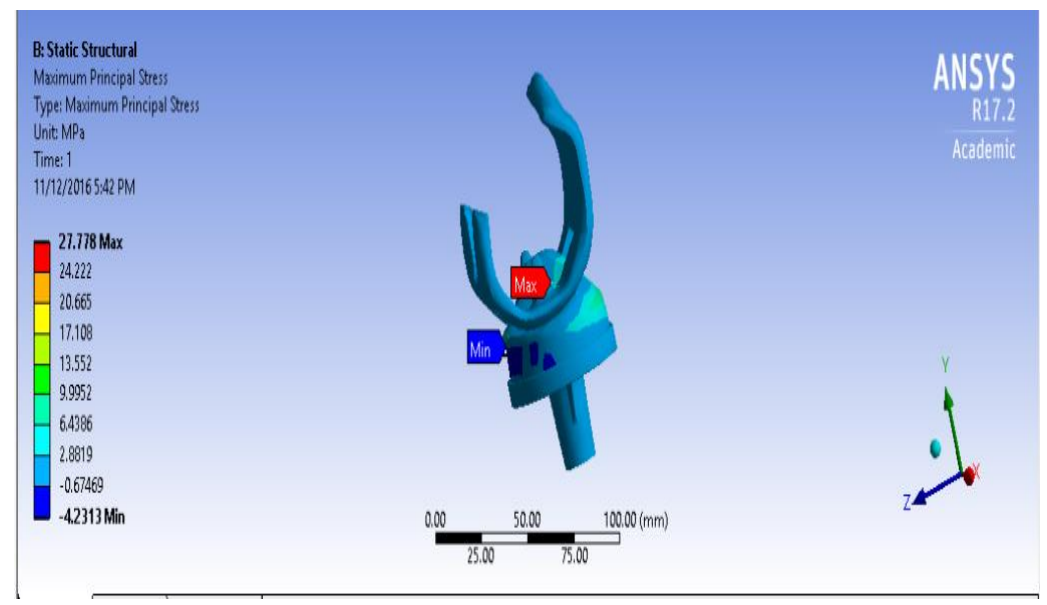

Fig. 4. Maximum Stress of $45^{\circ}$ of flexion angle which the value is almost the same with FEA result of Villa et. al [9]

The Finite Element Analysis is conducted by FEA Engineering Software which are well known method used in biomedical engineering [10-13]. The software used in this Finite Element Analysis is Ansys Workbench 17.0. This Ansys structural analysis software is suitable to solve complex structural engineering problems and make better, faster design decisions. With finite element analysis (FEA) tools, the simulations can be customized and automated, and parameterized them to analyse multiple design scenarios.

\section{Results and discussion}


The important of effect on flexion angle on contact stress also quite similar be proofed by Julian Favre et. al. via their research on "Modification of Knee Flexion Angle Has PatientSpecific Effects on Anterior Cruciate Ligament Injury Risk Factors During Jump Landing" [8]. Julian et. al. claimed that by increasing the knee flexion angle during jump landing may be an effective intervention to improve knee biomechanical risk factors associated with an ACL injury. While for this research, the aim is to improve the performance of the TKA in term of resistance to the fracture which subjected to different angle value of knee flexion. From the analysis, the results obtained from the structural analysis for this design shown that the maximum stress with the load applied at different flexion angle is better than the current one. By referred to the results of each design, the proposed design gave the better performance. The detailed of the results obtained from the analysis are recorded as in Table 1 below.

Table 1. Flexion angle, load apply and maximum stress between the current design and the proposed design.

\begin{tabular}{|c|c|c|c|l|c|}
\hline \multicolumn{2}{|l|}{ Verification based on previous journal [9] } & \multicolumn{3}{|c|}{ Proposed Design Results } \\
\hline $\begin{array}{l}\text { Flexion } \\
\text { Angle }\left[^{\circ}\right]\end{array}$ & $\begin{array}{l}\text { Force } \\
{[\mathrm{N}]}\end{array}$ & Max. Stress [MPa] & $\begin{array}{l}\text { Flexion } \\
\left.\text { Angle }{ }^{\circ}\right]\end{array}$ & $\begin{array}{l}\text { Force } \\
{[\mathrm{N}]}\end{array}$ & Max. Stress [MPa] \\
\hline $15^{\circ}$ & 2200 & 15 & $15^{\circ}$ & 2200 & 12.2 \\
\hline $45^{\circ}$ & 3200 & 27.7 & $45^{\circ}$ & 3200 & 23.6 \\
\hline $60^{\circ}$ & 2800 & 24.6 & $60^{\circ}$ & 2800 & 22.5 \\
\hline
\end{tabular}

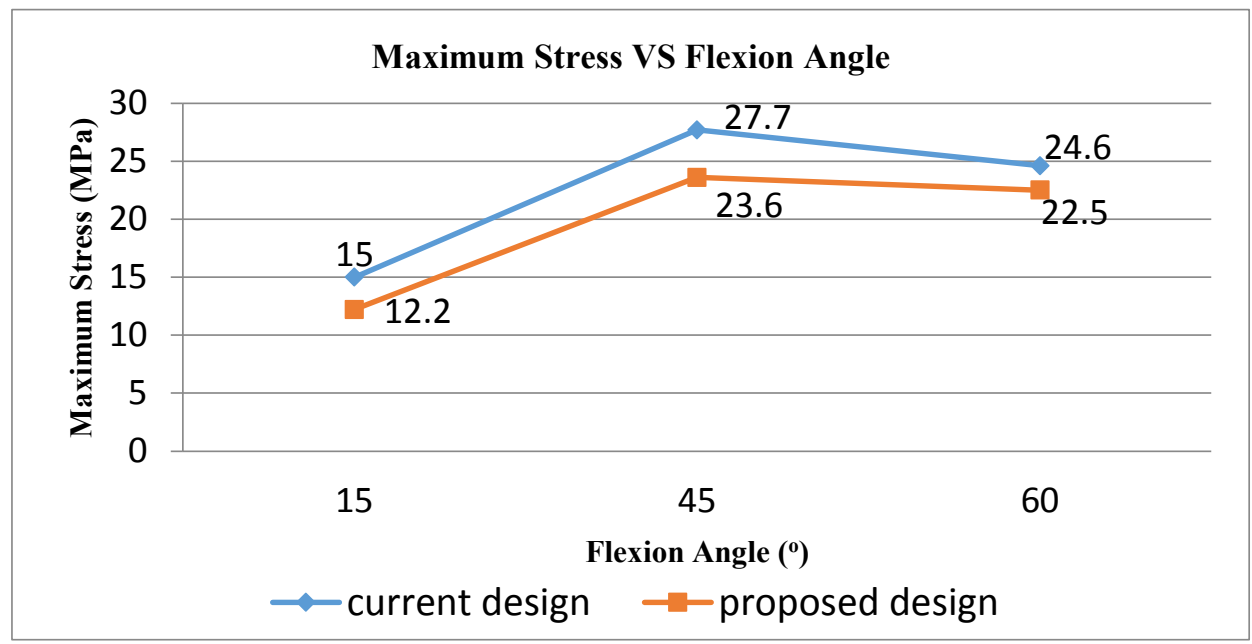

Fig. 5. Comparison between the performance of TKA of the current designs [9] and the proposed design (different flexion angles)

From the results obtained, the recorded value of the maximum stress from the different load applied on each design were then interpret into the graph form as shown in the Figure 5 above. The blue line on the graph represents current TKA (as reported by Villa et. al.) which the load applied are $2200 \mathrm{~N}$ at $15^{\circ}, 3200 \mathrm{~N}$ at $45^{\circ}$ and $2800 \mathrm{~N}$ at $60^{\circ}$ while the red one is for the proposed TKA design. By referred to the graph of current design shows the reading of the maximum stress are $15 \mathrm{MPa}$ at $15^{\circ}, 27.7 \mathrm{MPa}$ at $45^{\circ}$ and $24.6 \mathrm{MPa}$ at $60^{\circ}$. Whereas the 
maximum stress of the proposed design are 12.2 $\mathrm{MPa}, 23.6 \mathrm{MPa}$ and $22.5 \mathrm{Mpa}$ with flexion angle $15^{\circ}, 45^{\circ}$ and $60^{\circ}$ respectively.

At $15^{\circ}$ flexion, the FEA results show that the contact between femoral and tibia component is not yet present in the most its concave zone, so that the pressure distribution is quite uniform on the whole contact area and the maximum stress value is the lowest. The maximum stress of contact area at $45^{\circ}$ flexion become the highest values since the contact area seems to move from the posterior zone to the anterior one, corresponding to the most concave zone of the contact area. When talk about the comparison between maximum stress values of current design and proposed design of TKA, it is clearly shown in figure 5 that the value of maximum stress of proposed design is lower than current design while the maximum stress value pattern is still the same for both. The maximum stress values for proposed design are lower than current design of TKA due to the different of its design concept.

The main different in term of design concept is the contact surface between femoral and tibia components. The current design just allow small contact between femoral and tibia component during knee flexion while proposed design allow lot of contact area between both components. Thus, its gives more support to the load given during flexion which is can reduce the stress distribution. However, this new proposed design just better than current design in term of contact stress which is the main purposed of this study is to investigate the effect of flexion angles on contact stress. By other word, there are a lot of other criteria need to be considered in making the final design of the new proposed design of TKA to become better than the current design.

\section{Conclusions}

The main objective of this study has been achieved where the different flexion angle do gives an impact on the performance of the TKA. This study also successfully proof that proposed design of TKA is better than current design of TKA in term of contact stress for different flexion angles.

We would like to thank Universiti Malaysia Pahang through research grant RDU180395 for fully support the facilities and resources for this research.

\section{References}

1. Szivek, J. A. et al., The Journal of arthroplasty, 10, 480-491 (1995).

2. Andrew M. Kern, Donald D. Anderson, Journal of Biomechanics 48, 3427-3432 (2015).

3. Monan Wanga et al., Bioengineered 8, 105-112 (2017).

4. Jibanananda Satpathy et al., Journal of Hip Preservation Surgery 2, 287-294 (2015).

5. Rudert MJ et al., J Biomech Eng. 136, (2014).

6. Thor F.Besier et al. Patellofemoral joint contact area increases with knee flexion and weight-bearing. Journal of Orthopaedic Research 23, 345-350 (2005).

7. Jeffery T.Podraza et al., The Knee 17, 291-295 (2010).

8. Julien Favre, Caitlin Clancy, Ariel V. Dowling, Thomas P. Andriacchi, The American Journal of Sports Medicine, 44, 1540 - 1546 (2016)

9. Villa, T., et al., Journal of Biomechanics 37, 45-53 (2004).

10. Rosdi Daud et al., MATEC Web of Conferences 108, (2017).

11. Sandeep Kumar Parashar et al., Perspectives in Science 8, 696-698 (2016).

12. Mark Taylor et al., Journal of Biomechanics 48, 767-778 (2015). 
13. Rosdi Daud, S. Suaidah, H Mas-Ayu, S. H. Tomadi, M. S Salwani, A. Shah, Mohammed Rafiq Abdul-Kadir, Materials Science Forum, 916, 153-160 (2018) 